\title{
Quest for New Physics in Flavor Mixing and Dirac Neutrino Masses
}

\author{
Gui-Jun Ding \\ Interdisciplinary Center for Theoretical Study and Department of Modern Physics, \\ University of Science and Technology of China, Hefei, Anhui 230026, China
}

\begin{abstract}
We show that a unified description of the observed patterns of quark and lepton mixing can be achieved if the flavor group $D_{n}$ and CP symmetry are broken to $Z_{2} \times C P$ in the neutrino, charged lepton, up quark as well as down quark sectors, and the smallest group is $D_{14}$. We also perform a systematic study of how a dimension six operator $\bar{L} \widetilde{H} v_{R}\left(H^{+} H\right)$ for Dirac neutrino masses may occur at tree level and in one loop.
\end{abstract}

Keywords: Quark and lepton mixing, flavor symmetry, Dirac neutrinos

DOI: 10.31526/lhep.1.2019.120

\section{INTRODUCTION}

The experimental discovery of neutrino oscillation constitutes the first window into particle physics beyond the standard model (SM). The observation of neutrino oscillation implies that the neutrino has a non-zero mass, which requires a modification to the SM of particle physics. It is well-known that the quark and lepton flavor mixing are described by the CKM matrix and PMNS matrix respectively [1]. Neutrino and quark mixing parameters have been precisely measured. The global fit results for the magnitudes of all nine CKM elements are [1],

$\left|V_{C K M}\right|=\left(\begin{array}{ccc}0.97446 \pm 0.00010 & 0.22452 \pm 0.00044 & 0.00365 \pm 0.00012 \\ 0.22438 \pm 0.00044 & 0.97359_{-0.00011}^{+0.00010} & 0.04214 \pm 0.00076 \\ 0.00896_{-0.00023}^{+0.00024} & 0.04133 \pm 0.00074 & 0.999105 \pm 0.000032\end{array}\right)$

The $3 \sigma$ ranges on the magnitude of the elements of the leptonic mixing matrix [2] are given by:

$$
\left|U_{P M N S}\right|=\left(\begin{array}{lll}
0.797 \rightarrow 0.842 & 0.518 \rightarrow 0.585 & 0.143 \rightarrow 0.156 \\
0.233 \rightarrow 0.495 & 0.448 \rightarrow 0.679 & 0.639 \rightarrow 0.783 \\
0.287 \rightarrow 0.532 & 0.486 \rightarrow 0.706 & 0.604 \rightarrow 0.754
\end{array}\right) .
$$

Obviously the flavor mixing structures of quarks and leptons are very different from each other. Despite the overwhelming success of the standard model of particle physics, it does not shed any light on the understanding of the masses and mixings of quarks and leptons. The non-abelian discrete flavor symmetries have been widely studied to explain the leptonic mixing angles. In particular, it is found that the observed patterns of quark and lepton flavor mixing can be accommodated if the flavor and CP symmetries are broken to the residual symmetries $\mathrm{Z}_{2} \times C P$ in the charged lepton (up quark) and neutrino (down quark) sectors [3, 4]. The minimal flavor group is $\Delta(294)$ if the left-handed quarks and leptons are assigned to an irreducible triplet of flavor symmetry [3, 4]. In this work, we consider the dihedral flavor group $D_{n}$ with the symmetry breaking pattern $Z_{2} \times C P$, it is remarkable that $D_{14}$ is the smallest group to give a unified description of the quark and lepton mixing [5].

Non-vanishing neutrino masses definitely requires new physics beyond SM. Light neutrinos are frequently assumed to be Majorana particles in the literature. Given the absence of incontrovertible experimental signal for the existence of neutrinoless double beta decay, the possibility of Dirac neutrinos is still viable. The leading order operator for Dirac neutrino masses is $\bar{L} \widetilde{H} v_{R}$ in SM, and the smallness of neutrino masses requires the neutrino Yukawa coupling constants to be tiny. In this paper we shall classify and analyze the possible ways to generate Dirac neutrino mass through a dimension six operator $\bar{L} \widetilde{H} v_{R}\left(H^{\dagger} H\right)$.

The paper is organized as follows. In section 2, we show that both quark and lepton mixing patterns can be accommodated if the $D_{n}$ flavor group and CP symmetry are broken to $Z_{2} \times C P$ subgroups in the charged lepton, neutrino, up quark and down quark sectors. In section 3. we discuss all possible decompositions of the effective dimension six operator $\bar{L} \widetilde{H} v_{R}\left(H^{\dagger} H\right)$ for the Dirac neutrino masses at both tree level and one-loop level. Finally, summary and conclusions are given in section 4

\section{EXPLAINING LEPTON AND QUARK FLAVOR MIXING FROM DIHEDRAL FLAVOR GROUP AND CP SYMMETRY}

We first discuss the possible underlying symmetry of the quark and lepton flavor mixing, and the results are based on [5]. The dihedral group $D_{n}$ is the symmetry group of an $n$-sided regular polygon. Since a regular polygon with $n$ sides has $2 n$ different symmetries: $n$ rotational symmetries and $n$ reflection symmetries, the group order of $D_{n}$ is $2 n$. All the group elements of $D_{n}$ can be generated by two generators $R$ and $S$ which satisfy,

$$
R^{n}=S^{2}=(R S)^{2}=1,
$$

where $R$ refers to the rotation and $S$ is the reflection. The group elements of $D_{n}$ can be expressed as

$$
g=S^{\alpha} R^{\beta}
$$

where $\alpha=0,1$ and $\beta=0,1, \ldots, n-1$. The irreducible presentations of $D_{n}$ are one-dimensional and two-dimensional. If the index $n$ is an odd integer, $D_{n}$ has two singlet representations and $\frac{n-1}{2}$ doublet representations. For $n$ being even, $D_{n}$ has four singlet representations and $\frac{n}{2}-1$ doublet representations. We denote the one-dimensional representations with $\mathbf{1}_{i}$ and the two-dimensional ones with $\mathbf{2}_{j}$. The generators $R$ and $S$ for the one-dimensional representations are given by

$$
\begin{array}{ll}
\mathbf{1}_{1}: R=S=1, & \mathbf{1}_{2}: R=1, S=-1, \\
\mathbf{1}_{3}: R=-1, S=1, & \mathbf{1}_{4}: R=S=-1,
\end{array}
$$

where $\mathbf{1}_{3}$ and $\mathbf{1}_{4}$ exist only for even $n$. In the doublet representations, we have

$$
\mathbf{2}_{j}: R=\left(\begin{array}{cc}
e^{2 \pi i \frac{j}{n}} & 0 \\
0 & e^{-2 \pi i \frac{j}{n}}
\end{array}\right), \quad S=\left(\begin{array}{ll}
0 & 1 \\
1 & 0
\end{array}\right),
$$


with $j=1, \ldots, \frac{n-1}{2}$ for odd $n$ and $j=1, \ldots, \frac{n}{2}-1$ for even $n$.

In this work, we shall extend the dihedral flavor group to involve $\mathrm{CP}$ also as symmetry. Note that the $D_{3}$ group in combination with $\mathrm{CP}$ has been studied in [6]. The $\mathrm{CP}$ transformation is strongly constrained by the requirement that the subsequent application of the CP transformation, the flavor symmetry and the $\mathrm{CP}$ transformation should be another element of the flavor group [7, 8, 9, 10], i.e.

$$
X_{\mathbf{r}} \rho_{\mathbf{r}}^{*}(g) X_{\mathbf{r}}^{\dagger}=\rho_{\mathbf{r}}\left(g^{\prime}\right), \quad g, g^{\prime} \in G_{f},
$$

where $\rho_{\mathbf{r}}(g)$ is the representation matrix of the element $g$ in the representation $\mathbf{r}, g$ and $g^{\prime}$ are in general different, and $X_{\mathbf{r}}$ is the $\mathrm{CP}$ transformation. Solving the consistency condition of Eq. (7), we find that the CP transformations compatible with $D_{n}$ are of the same form as the flavor symmetry transformations in our working basis, i.e.

$$
X_{\mathbf{r}}=\rho_{\mathbf{r}}(g), \quad g \in D_{n} .
$$

Without loss of generality, the three generations of left-handed lepton and quark doublets are assigned to transform as a direct sum of singlet $\mathbf{1}_{1}$ and doublet $\mathbf{2}_{1}$ representations of $D_{n}$,

$$
L \sim\left(\mathbf{1}_{1}, \mathbf{2}_{1}\right), \quad Q \sim\left(\mathbf{1}_{1}, \mathbf{2}_{1}\right) .
$$

We assume that the flavor group $D_{n}$ and CP symmetry are broken to $Z_{2}^{g_{e}} \times X_{e}$ and $Z_{2}^{g_{v}} \times X_{v}$ in the charged lepton and neutrino sectors respectively. The charged lepton and neutrino mass matrices invariant under these residual symmetries would be diagonalized by the following unitary transformations [11, 3, 4, 12]

$$
U_{e}=\Sigma_{e} S_{23}\left(\theta_{e}\right), \quad U_{v}=\Sigma_{v} S_{23}\left(\theta_{v}\right) Q_{v}^{\dagger},
$$

up to independent column permutations. Here $\Sigma_{e}$ and $\Sigma_{v}$ are the Takagi factorizations for $X_{e}$ and $X_{v}$ respectively and they satisfy

$$
\begin{array}{ll}
X_{e}=\Sigma_{e} \Sigma_{e}^{T}, & \Sigma_{e}^{\dagger} \rho\left(g_{e}\right) \Sigma_{e}= \pm \operatorname{diag}(1,-1,-1), \\
X_{v}=\Sigma_{v} \Sigma_{v}^{T}, & \Sigma_{v}^{\dagger} \rho\left(g_{v}\right) \Sigma_{v}= \pm \operatorname{diag}(1,-1,-1) .
\end{array}
$$

The symbol $S_{23}(\theta)$ in Eq. 10 denotes a rotation in the (23)plane through an angle $\theta$,

$$
S_{23}(\theta)=\left(\begin{array}{ccc}
1 & 0 & 0 \\
0 & \cos \theta & \sin \theta \\
0 & -\sin \theta & \cos \theta
\end{array}\right)
$$

where the fundamental region of the rotation angle $\theta$ is $[0, \pi)$. For Majorana neutrinos, $Q_{v}$ is a diagonal matrix with elements \pm 1 and $\pm i$ to ensure positiveness of the light neutrino masses, and it can be parametrized as

$$
Q_{v}=\operatorname{diag}\left(1, i^{k_{1}}, i^{k_{2}}\right),
$$

with $k_{1,2}=0,1,2,3$. As a consequence, the residual symmetry enforces the lepton mixing matrix to be of the form [11, 3, 5]

$$
U \equiv U_{e}^{\dagger} U_{v}=S_{23}^{T}\left(\theta_{e}\right) \Sigma_{e}^{\dagger} \Sigma_{v} S_{23}\left(\theta_{v}\right) Q_{v}^{\dagger},
$$

up to permutations of rows and columns. We see that the mixing matrix as well as mixing angles and $\mathrm{CP}$ phases only depend on two real rotation angles $\theta_{e}$ and $\theta_{v}$ in the range between 0 and $\pi$. Analogously if the residual symmetries $Z_{2}^{g_{u}} \times X_{u}$ and $Z_{2}^{g_{d}} \times X_{d}$ are preserved by the up quark and down quark mass matrices respectively, the CKM mixing matrix would be

$$
V=S_{23}^{T}\left(\theta_{u}\right) \Sigma_{u}^{\dagger} \Sigma_{d} S_{23}\left(\theta_{d}\right),
$$

up to possible rows and columns permutations are omitted, $\Sigma_{u}$ and $\Sigma_{d}$ are the Takagi factorizations for $X_{u}$ and $X_{d}$, respectively and the identities similar to Eq. (11) should be fulfilled. Considering all possible residual subgroups from $D_{n}$ and CP symmetry, we find that the hierarchical CKM mixing matrix can only be accommodated for $g_{u}=S R^{x}, X_{u}=S R^{n / 2}, g_{d}=S R^{y}$ and $X_{d}=S$ with $x, y=0,1, \ldots, n-1$. The quark mixing matrix up to permutations of rows and columns is determined to be

$$
V=\left(\begin{array}{ccc}
\cos \varphi & -c_{d} \sin \varphi & s_{d} \sin \varphi \\
c_{u} \sin \varphi & c_{d} c_{u} \cos \varphi+i s_{d} s_{u} & -s_{d} c_{u} \cos \varphi+i c_{d} s_{u} \\
-s_{u} \sin \varphi & -c_{d} s_{u} \cos \varphi+i s_{d} c_{u} & s_{d} s_{u} \cos \varphi+i c_{d} c_{u}
\end{array}\right)
$$

where $s_{u}=\sin \theta_{u}, s_{d}=\sin \theta_{d}, c_{u}=\cos \theta_{u}, c_{d}=\cos \theta_{d}$, and the parameter $\varphi$ is fixed by residual symemtry

$$
\varphi=\frac{y-x}{n} \pi .
$$

Taking into account all possible permutations of rows and columns, following nine mixing patterns can be obtained,

$$
\begin{array}{lll}
V_{1}=V, & V_{2}=V P_{12}, & V_{3}=V P_{13}, \\
V_{4}=P_{12} V, & V_{5}=P_{12} V P_{12}, & V_{6}=P_{12} V P_{13}, \\
V_{7}=P_{23} P_{12} V, & V_{8}=P_{23} P_{12} V P_{12}, & V_{9}=P_{23} P_{12} V P_{13},
\end{array}
$$

with the permutation matrices

$$
P_{12}=\left(\begin{array}{lll}
0 & 1 & 0 \\
1 & 0 & 0 \\
0 & 0 & 1
\end{array}\right), P_{13}=\left(\begin{array}{lll}
0 & 0 & 1 \\
0 & 1 & 0 \\
1 & 0 & 0
\end{array}\right), P_{23}=\left(\begin{array}{lll}
1 & 0 & 0 \\
0 & 0 & 1 \\
0 & 1 & 0
\end{array}\right) \text {. }
$$

For each group index $n$ and the possible values of $\varphi$, we have numerically analyzed whether the quark mixing angles and $C P$ violation phase can be reasonably close to their measured values for certain values of the free parameters $\theta_{u, d}$. We find that the experimental data on CKM mixing matrix can be described by the two mixing patterns $V_{1}$ and $V_{2}$, and the minimal flavor group is $D_{14}$.

For both mixing patterns $V_{1}$ and $V_{2}$, the three quark mixing angles $\theta_{12}^{q}, \theta_{13}^{q}, \theta_{23}^{q}$ and one CP phase $\delta_{C P}^{q}$ depend on two free parameters $\theta_{u}$ and $\theta_{d}$. Therefore two sum rules among the mixing parameters can be obtained,

$$
\begin{aligned}
& \sin \delta_{C P}^{q} \simeq \frac{\sin 2 \varphi}{\sin 2 \theta_{12}^{q} \cos ^{2} \theta_{13}^{q} \cos \theta_{23}^{q},} \\
& \cos ^{2} \theta_{13}^{q} \cos ^{2} \theta_{12}^{q}=\cos ^{2} \varphi, \text { for } V_{1}, \\
& \cos ^{2} \theta_{13}^{q} \sin ^{2} \theta_{12}^{q}=\cos ^{2} \varphi, \text { for } V_{2} .
\end{aligned}
$$

It is notable that good agreement with experimental data can be achieved for $V_{1}$ with $\varphi=\pi / 14$ and $V_{2}$ with $\varphi=3 \pi / 7$, and we give two numerical benchmark examples,

$$
\begin{aligned}
& V_{1}:\left\{\begin{array}{c}
\varphi=\pi / 14, \quad \theta_{u}=0.01237 \pi, \quad \theta_{d}=0.99473 \pi, \\
\sin \theta_{12}^{q}=0.22249, \quad \sin \theta_{13}^{q}=0.00369, \\
\sin \theta_{23}^{q}=0.04206, \quad J_{C P}^{q}=3.104 \times 10^{-5},
\end{array}\right. \\
& V_{2}:\left\{\begin{array}{c}
\varphi=3 \pi / 7, \quad \theta_{u}=0.01326 \pi, \quad \theta_{d}=0.00117 \pi, \\
\sin \theta_{12}^{q}=0.22252, \quad \sin \theta_{13}^{q}=0.00357, \\
\sin \theta_{23}^{q}=0.04166, \quad J_{C P}^{q}=3.223 \times 10^{-5},
\end{array}\right.
\end{aligned}
$$


which are compared with the observed values [13]

$$
\begin{array}{ll}
\sin \theta_{12}^{q}=0.22500 \pm 0.00100, & \sin \theta_{13}^{q}=0.003675 \pm 0.000095, \\
\sin \theta_{23}^{q}=0.04200 \pm 0.00059, & J_{C P}^{q}=(3.120 \pm 0.090) \times 10^{-5} .
\end{array}
$$

In a similar way, the experimentally measured values of the lepton mixing angles can be accommodated if the residual symmetries of the charged lepton and neutrino sectors are $g_{v}=S R^{y}, X_{v}=S, g_{e}=S R^{x}$ and $X_{e}=S R^{n / 2}$ with $x, y=$ $0,1, \ldots, n-1$. The lepton mixing matrix would be of the same form as Eq. 16, and the possible permutations of rows and columns can give rise to nine mixing patterns $U_{i}(i=1, \ldots, 9)$. The mixing matrix $U_{i}$ can be obtained from $V_{i}$ by substituting $\theta_{u}, \theta_{d}$ with $\theta_{e}, \theta_{v}$ respectively. For the $D_{14}$ flavor group and the residual symmetry indices $y=4, x=0$ which imply $\varphi=2 \pi / 7$, the mixing pattern $U_{9}$ can give experimentally favored values of the PMNS matrix, and the best fit values of the mixing parameters are

$$
\begin{aligned}
& \theta_{e}^{\mathrm{bf}}=0.439 \pi, \quad \theta_{v}^{\mathrm{bf}}=0.814 \pi, \quad \chi_{\min }^{2}=1.841, \\
& \sin ^{2} \theta_{13}=0.0224, \quad \sin ^{2} \theta_{12}=0.310, \\
& \sin ^{2} \theta_{23}=0.602, \quad \delta_{C P} / \pi=1.532, \\
& \alpha_{21} / \pi=0.167(\bmod 1), \quad \alpha_{31} / \pi=0.116(\bmod 1) .
\end{aligned}
$$

In short, the $D_{14}$ group in combination with CP symmetry allows for a unified description of quark and lepton mixing, and it provides a new starting point for building models of quark and lepton mixing.

\section{GENERATION OF DIRAC NEUTRINO MASSES}

In this section, we proceed to discuss the possible new physics in Dirac neutrino mass generation. The results are already published in [14]. The signal of neutrinoless double beta decay has not been observed, the nature of neutrinos is still unclear. Although usually neutrinos are assumed to be Majorana particles, and we can not exclude the possibility of Dirac neutrinos at present. In the context of standard model, the most general effective operators for the Dirac neutrino masses take the following form

$$
\mathscr{L}_{4+2 n}^{D}=-y_{\alpha \beta} \bar{L}_{\alpha} \widetilde{H} v_{R \beta}\left(\frac{H^{\dagger} H}{\Lambda^{2}}\right)^{n}+\text { H.c. },
$$

where $L=\left(v_{L}, l_{L}\right)^{T}$ is the left-handed lepton doublet, $H=$ $\left(H^{+}, H^{0}\right)^{T}$ is the Higgs doublet with $\widetilde{H}=i \sigma_{2} H^{*}$, and $v_{R}$ denote the right-handed neutrino fields. Since $v_{R}$ are SM singlets, the Majorana mass term $m_{N} \overline{v_{R}^{c}} v_{R}$ is allowed such that the light neutrinos would be Majorana particles. In order to realize Dirac neutrinos, additional symmetry is generally needed to forbid this right-handed neutrino Majorana mass term and it is usually taken to be $U(1)_{L}$ lepton number. Obviously the lowest dimensional operator is $-y_{\alpha \beta} \bar{L}_{\alpha} \widetilde{H} v_{R \beta}$ for $n=0$ and it generates the neutrino mass after electroweak symmetry breaking. The smallness of neutrino masses require the coupling constant be tiny $y_{\alpha \beta} \sim \mathcal{O}\left(10^{-11}\right)$. This renormalizable operator can also be generated at one loop, and the messenger fields should be quite heavy to give a tiny $y_{\alpha \beta}$.

We shall focus on the dimension six operator of the Dirac neutrino masses corresponding to $n=1$, this nonrenormalizable operator can be induced at either tree level or loop level [14, 15]. Moreover, renormalizability fixes possible vertices to only dimension-four three and four point vertices. The trilinear couplings can involve two, one or none SM fields, i.e. $\bar{F} L H, \bar{F} L \widetilde{H}, \bar{F} v_{R} H, \bar{F} v_{R} \widetilde{H}, \bar{L} v_{R} S, \bar{F} L S, \bar{F} v_{R} S, \overline{F_{1}} F_{2} H, \bar{F}_{1} F_{2} \widetilde{H}$ and $\bar{F}_{1} F_{2} S$ where $F$ and $S$ denote fermion and scalar beyond $\mathrm{SM}$. For each possible three point vertex, the $S U(2) \times U(1)_{Y}$ gauge invariance requires,

$$
\begin{aligned}
& \bar{F} L H: n_{F}=1,3, \quad Y_{F}=0, \\
& \bar{F} L \tilde{H}: n_{F}=1,3, \quad Y_{F}=-2, \\
& \bar{F} v_{R} H: n_{F}=2, \quad Y_{F}=1, \\
& \bar{F} v_{R} \widetilde{H}: n_{F}=2, \quad Y_{F}=-1, \\
& \bar{L} v_{R} S: n_{S}=2, \quad Y_{S}=-1, \\
& \bar{F} L S: n_{F} \otimes n_{S} \supset 2, \quad Y_{S}-Y_{F}=1, \\
& \bar{F} v_{R} S: n_{F}=n_{S}, \quad Y_{F}=Y_{S}, \\
& \bar{F}_{1} F_{2} H: n_{F_{1}} \otimes n_{F_{2}} \supset 2, \quad Y_{F_{1}}-Y_{F_{2}}=1, \\
& \bar{F}_{1} F_{2} \widetilde{H}: n_{F_{1}} \otimes n_{F_{2}} \supset 2, \quad Y_{F_{2}}-Y_{F_{1}}=1, \\
& \bar{F}_{1} F_{2} S: n_{F_{1}} \otimes n_{F_{2}} \supset n_{S}, \quad Y_{F_{2}}-Y_{F_{1}}+Y_{S}=0,
\end{aligned}
$$

where $n_{X}$ corresponds to the $S U(2)$ representation under which the $X$ field transforms, and $Y_{X}$ refers to the $U(1)_{Y}$ charge of the $X$ field. The four point interaction vertex must involve four scalar fields, and three, two, one or none of them can be SM Higgs fields. Explicitly they can be $H H H S_{1}, H H \widetilde{H} S_{1}, H \widetilde{H} \widetilde{H} S_{1}$, $\widetilde{H} \widetilde{H} \widetilde{H} S_{1}, H H S_{1} S_{2}, H \widetilde{H} S_{1} S_{2}, \widetilde{H} \widetilde{H} S_{1} S_{2}, H S_{1} S_{2} S_{3}, \widetilde{H} S_{1} S_{2} S_{3}$ and $S_{1} S_{2} S_{3} S_{4}$. In this case, the SM gauge invariance implies,

$$
\begin{aligned}
& H H H S_{1}: n_{S_{1}}=4, \quad Y_{S_{1}}=-3, \\
& H H \tilde{H} S_{1}: n_{S_{1}}=2,4, \quad Y_{S_{1}}=-1, \\
& H \widetilde{H} \widetilde{H} S_{1}: n_{S_{1}}=2,4, \quad Y_{S_{1}}=1, \\
& \widetilde{H} \widetilde{H} S_{1}: n_{S_{1}}=4, \quad Y_{S_{1}}=3, \\
& H H S_{1} S_{2}: n_{S_{1}} \otimes n_{S_{2}} \supset 3, \quad Y_{S_{1}}+Y_{S_{2}}=-2, \\
& H \widetilde{H} S_{1} S_{2}: n_{S_{1}} \otimes n_{S_{2}} \supset 1,3, \quad Y_{S_{1}}+Y_{S_{2}}=0, \\
& \widetilde{H} \widetilde{H} S_{1} S_{2}: n_{S_{1}} \otimes n_{S_{2}} \supset 3, \quad Y_{S_{1}}+Y_{S_{2}}=2, \\
& H S_{1} S_{2} S_{3}: n_{S_{1}} \otimes n_{S_{2}} \otimes n_{S_{3}} \supset 2, \quad Y_{S_{1}}+Y_{S_{2}}+Y_{S_{3}}=-1, \\
& \widetilde{H} S_{1} S_{2} S_{3}: n_{S_{1}} \otimes n_{S_{2}} \otimes n_{S_{3}} \supset 2, \quad Y_{S_{1}}+Y_{S_{2}}+Y_{S_{3}}=1, \\
& S_{1} S_{2} S_{3} S_{4}: n_{S_{1}} \otimes n_{S_{2}} \otimes n_{S_{3}} \otimes n_{S_{4}} \supset 1, Y_{S_{1}}+Y_{S_{2}}+Y_{S_{3}}+Y_{S_{4}}=0 .(27)
\end{aligned}
$$

Similar to the well-known seesaw models, we find that there are only two possible topologies named as F1 and F2 at tree level, as shown in Table 1 . Then we specify the fermion and scalar internal lines as well as the external lines of each topology, the quantum numbers of the messenger fields are determined from the invariance of each interaction vertex under the SM gauge group. The classification of what particles can induce this $d=6$ operator $\bar{L} \widetilde{H} v_{R}\left(H^{\dagger} H\right)$ at tree level is summarized in Table 1 The transformation properties of the new fields are denoted by the notation $n_{Y}^{\mathcal{L}}$, where $n$ refers to the $S U(2)$ transformation ( $\mathbf{1}$ for singlet, $\mathbf{2}$ for doublet, and $\mathbf{3}$ for triplet), $Y$ is its hypercharge, and $\mathcal{L}$ represents the Lorentz nature and it can be either $S$ for scalar or $F$ for fermion. We assume new fermions are vector-like to ensure anomaly cancellation. The diagrams with scalar and vector bosons are equivalent, and the resulting neutrino masses for the diagrams with vectors can be straightforwardly obtained from those of the diagrams with scalars. In addition, vector bosons are generally the gauge bosons of a certain gauge symmetry, their mass are generated via the spontaneous breaking of that symmetry. As a result, the scalar sector 


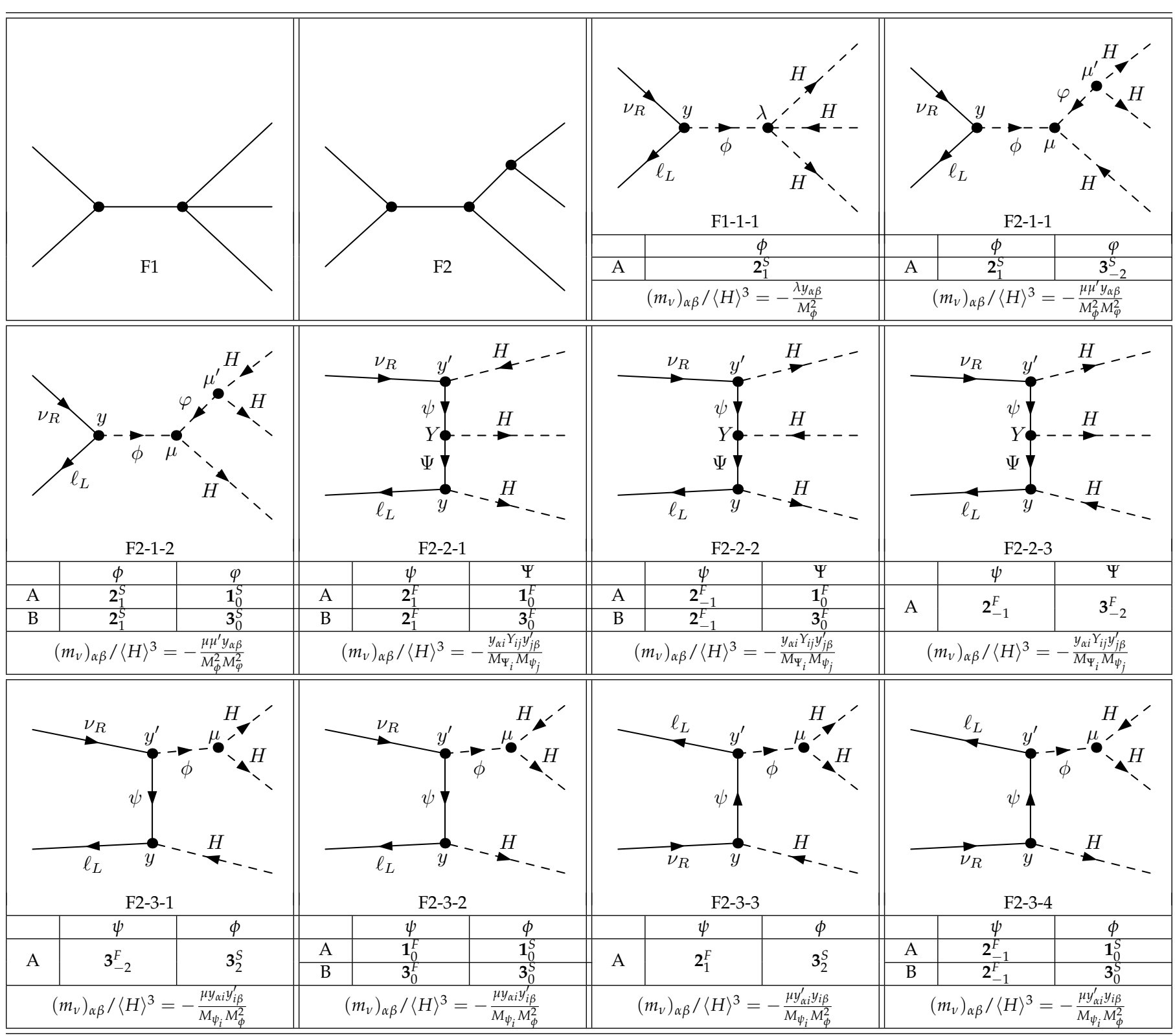

TABLE 1: Possible topologies and diagrams for the tree level decomposition of the dimension six effective operator $\bar{L} \widetilde{H} v_{R}\left(H^{\dagger} H\right)$. This table is taken from [14].

of these models should be discussed carefully as well, the corresponding analysis is highly model dependent. Hence we shall consider the scalar and fermion mediated models here.

Following the diagram-based approach of Refs. [16, 17], we can find out all possible one-loop realizations for this dimension six Dirac neutrino mass operator. Firstly, we use the program FeynArts [18] to construct the one-loop topologies with five external legs, the self-energy and tadpole diagrams are excluded. It turns out that there are totally 16 distinct topologies. Then we specify the Lorentz nature (spinor or scalar) of each line. For each topology, we can use FeynArts to find out all possible scalar or fermionic assignments for lines. We denote the fermions with solid line, and scalars with dashed line. There are usually more than one possibilities of assigning the five external legs to the lepton doublet $L$, the right-handed neutrino singlet $v_{R}$, two Higgs doublets $H$ and the Higgs conjugate $H^{\dagger}$, therefore a given topology can give rise to several Feynman diagrams. All possible Feynman diagrams are generated with the help of FeynArts.

All the Feynman diagrams can be divided into two categories: the diagrams which lead to finite loop integrals and the ones which involve infinite loop integrals. Since the divergence can be absorbed by the counter terms of the tree level realizations of this dimension six operator, we are concerned with the diagrams with finite loop integrals. Eventually we find five diagrams T2-2, T3-6, T8-3, T8-4 and T8-5 displayed in Figure 1 for which both the tree level realizations of $d=6$ operator and one-loop diagrams of $d=4$ operator can be avoided for certain assignments of the mediators. The quantum numbers of the mediator fields can be fixed from the requirement of gauge invariance in Eqs. 26, 27). Similar to the scotogenic model for Majorana neutrinos 119, the new messenger fields in the above 


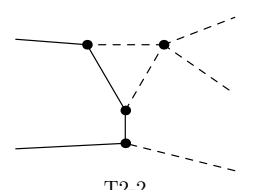

$\mathrm{T} 2-2$

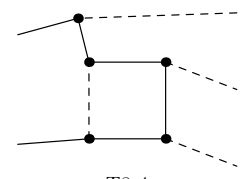

T8-4
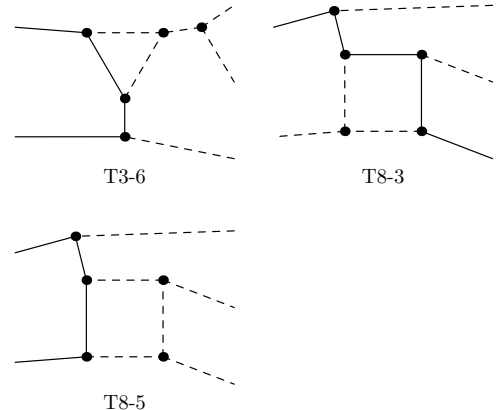

FIGURE 1: Finite one-loop diagrams for the $d=6$ Dirac neutrino mass operator $\bar{L} \widetilde{H} v_{R}\left(H^{\dagger} H\right)$, where the dashed lines denote scalars and the solid lines denote fermions. The lower order contributions can be absent for certain quantum numbers of the messengers in these diagrams [14].

one-loop diagrams could possibly be identified as dark matter particles such that neutrino masses and dark matter can be accounted for in a single model, please see Ref. [14] for details. Before closing this section, we note that the analysis of section 2 is independent of the neutrino mass generation mechanism. If the neutrinos are Dirac particles and the mass term preserves the residual symmetry $Z_{2} \times C P$ shown in section 2 , the same viable lepton mixing pattern can be achieved as well.

\section{CONCLUSION}

This paper is based on the talk in the conference "The quest for new physics, 12-14 December 2018, Casa de la Ciencia del CSIC". The origin of the masses and mixings of quarks and leptons is a fundamental problem in particle physics. Neutrino oscillation and the existence of neutrino mass provide a guide for the nature of the new physics beyond SM. It turns out that broken flavor symmetry based on the discrete groups is particularly suitable to describe the three lepton mixing angles. A recent progress is to extend the discrete flavor symmetry by including the $\mathrm{CP}$ symmetry, it allows to predict both lepton mixing angles and $C P$ violation phases in terms of few free parameters. The dihedral group $D_{n}$ as flavor symmetry and the interplay with CP symmetry are discussed in this work. The three generations of left-handed lepton and quark fields are assigned to a direct sum of the one-dimensional representation $\mathbf{1}_{1}$ and two-dimensional representation $2_{1}$ of $D_{n}$, this is in contrast with the usual irreducible triplet assignments. The experimentally measured values of the CKM and PMNS mixing matrices can be obtained if the flavor group $D_{n}$ and CP symmetry are broken down to $Z_{2} \times C P$ subgroups in the charged lepton, neutrino, up quark and down quark sectors, and minimal group is $D_{14}$. New physics beyond SM is necessary to generate the tiny neutrino masses, and the underlying physics are different for Majorana neutrinos and Dirac neutrinos. A systematical classification of all possible tree and one-loop realizations for a $d=6$ Dirac neutrino mass operator $\bar{L} \widetilde{H} v_{R}\left(H^{\dagger} H\right)$ is performed here.

\section{ACKNOWLEDGEMENT}

This work is supported by the National Natural Science Foundation of China under Grant Nos.11522546 and 11835013.
G.-J.D. acknowledges the hospitality of the Astroparticle and High Energy Physics Group, IFIC where part of this work was carried out.

\section{References}

[1] Particle Data Group Collaboration, M. Tanabashi et al., "Review of Particle Physics," Phys. Rev. D98 no. 3, (2018) 030001

[2] I. Esteban, M. C. Gonzalez-Garcia, A. Hernandez-Cabezudo, M. Maltoni, and T. Schwetz, "Global analysis of three-flavor neutrino oscillations: synergies and tensions in the determination of $\theta_{23}, \delta_{C P}$, and the mass ordering," arXiv:1811.05487 [hep-ph]

[3] C.-C. Li, J.-N. Lu, and G.-J. Ding, "Toward a unified interpretation of quark and lepton mixing from flavor and CP symmetries," JHEP 02 (2018) 038. arXiv:1706.04576 [hep-ph]

[4] J.-N. Lu and G.-J. Ding, "Quark and lepton mixing patterns from a common discrete flavor symmetry with a generalized CP symmetry," Phys. Rev. D98 no. 5, (2018) 055011. arXiv: 1806.02301 [hep-ph]

[5] J.-N. Lu and G.-J. Ding, "Dihedral flavor group as the key to understand quark and lepton flavor mixing," arXiv:1901.07414 [hep-ph]

[6] E. Ma, "Cobimaximal neutrino mixing from $S_{3} \times Z_{2}$," Phys. Lett. B777 (2018) 332-334 arXiv: 1707.03352 [hep-ph]

[7] W. Grimus and M. N. Rebelo, "Automorphisms in gauge theories and the definition of CP and P," Phys. Rept. 281 (1997) 239-308, arXiv:hep-ph/9506272 [hep-ph].

[8] F. Feruglio, C. Hagedorn, and R. Ziegler, "Lepton Mixing Parameters from Discrete and CP Symmetries," JHEP 07 (2013) 027, arXiv:1211.5560 [hep-ph].

[9] M. Holthausen, M. Lindner, and M. A. Schmidt, "CP and Discrete Flavour Symmetries," JHEP 04 (2013) 122. arXiv:1211.6953 [hep-ph]

[10] M.-C. Chen, M. Fallbacher, K. T. Mahanthappa, M. Ratz, and A. Trautner, "CP Violation from Finite Groups," Nucl. Phys. B883 (2014) 267-305, arXiv: 1402.0507 [hep-ph]

[11] J.-N. Lu and G.-J. Ding, "Alternative Schemes of Predicting Lepton Mixing Parameters from Discrete Flavor and CP Symmetry," Phys. Rev. D95 no. 1, (2017) 015012, arXiv:1610.05682 [hep-ph]

[12] C.-Y. Yao and G.-J. Ding, "CP Symmetry and Lepton Mixing from a Scan of Finite Discrete Groups," Phys. Rev. D94 no. 7, (2016) 073006 arXiv:1606.05610 [hep-ph]

[13] The results of the global data fitting from the UTfit Collaboration. http://www.utfit.org/UTfit/ResultsSummer2018SM

[14] C.-Y. Yao and G.-J. Ding, "Systematic Study of One-Loop Dirac Neutrino Masses and Viable Dark Matter Candidates," Phys. Rev. D96 no. 9, (2017) 095004. arXiv:1707.09786 [hep-ph]

[15] S. Centelles Chuli, R. Srivastava, and J. W. F. Valle, "Seesaw Dirac neutrino mass through dimension-six operators," Phys. Rev. D98 no. 3, (2018) 035009. arXiv:1804.03181 [hep-ph]

[16] F. Bonnet, M. Hirsch, T. Ota, and W. Winter, "Systematic study of the $\mathrm{d}=5$ Weinberg operator at one-loop order," JHEP 07 (2012) 153, arXiv: 1204.5862 [hep-ph] 
[17] D. Aristizabal Sierra, A. Degee, L. Dorame, and M. Hirsch, "Systematic classification of two-loop realizations of the Weinberg operator," JHEP 03 (2015) 040, arXiv: 1411.7038 [hep-ph]

[18] T. Hahn, "Generating Feynman diagrams and amplitudes with FeynArts 3," Comput. Phys. Commun. 140 (2001) 418-431, arXiv:hep-ph/0012260 [hep-ph]
[19] E. Ma, "Verifiable radiative seesaw mechanism of neutrino mass and dark matter," Phys. Rev. D73 (2006) 077301, arXiv:hep-ph/0601225 [hep-ph] 